\title{
Stochastic assessment of distributed generation hosting capacity and energy efficiency in active distribution networks
}

ISSN 1751-8687

Received on 9th April 2017

Revised 5th July 2017

Accepted on 20th July 2017

E-First on 5th October 2017

doi: 10.1049/iet-gtd.2017.0557

www.ietdl.org

\author{
Darwin Alexis Quijano1,2凶, Jianhui Wang ${ }^{1}$, Mushfiqur R. Sarker ${ }^{1}$, Antonio Padilha-Feltrin ${ }^{2}$ \\ ${ }^{1}$ Energy Systems Division, Argonne National Laboratory, Argonne, IL, USA \\ ${ }^{2}$ Department of Electrical Engineering, Universidade Estadual Paulista - UNESP, Ilha Solteira, São Paulo, Brazil \\ 凶E-mail: alexisqr@yahoo.es
}

\begin{abstract}
Active network management (ANM) aims to increase the capacity of variable distributed generation (DG), which can be connected to existing distribution networks. In this study, it is proposed to simultaneously consider the efficient use of energy resources when high shares of DG are procured through the ANM approach. To that end, a multi-period and multiobjective optimisation algorithm, based on the linearised optimal power flow, is formulated. The algorithm seeks to maximise the installed capacity of DG while minimising the energy losses and consumption of voltage-dependent loads. The objectives are optimised considering the coordinated operation of voltage regulators and on-load tap changers, and the management of DG generation curtailment and reactive power compensation from DG. Additionally, the effects of load and generation uncertainties are addressed through a two-stage stochastic programming formulation of the multiobjective problem. The result is a set of noninferior solutions, which allows exploring the degree of conflict among the objectives. The proposed approach was tested on two IEEE test feeders and the solutions show a significant improvement in the system's energy efficiency with a low impact on the amount of connected DG.
\end{abstract}

\section{Nomenclature}

Sets

$\Omega_{b} \quad$ set of network nodes

$\Omega_{l} \quad$ set of lines

$T \quad$ set of discrete time periods

$R \quad$ set of buses with voltage regulation devices

$G$ set of candidate nodes for DG allocation

$S \quad$ set of scenarios

$M \quad$ set of line segments approximating the square of the power flows in lines

$U$ set of line segments approximating the capability curve of generators and the substation transformer capacity

\section{Parameters}

$r_{i, j} / x_{i, j} \quad$ resistance/reactance of the line segment between

$P l_{i}^{Z}$

$P l_{i}^{I}$

$P l_{i}^{P}$

$\zeta_{t}^{Z}$

$\zeta_{t}^{I}$

$\zeta_{t}^{P}$

$\omega_{t}$

$\gamma_{\min }^{c} / V_{\max }$

$\Delta \operatorname{tap}_{i}$

$\operatorname{tap}_{i}^{\min } / \operatorname{tap}_{i}^{\max }$

$S_{1, \max }$

$\mathrm{TAP}_{i}^{\max }$ nodes $i$ and $j$

peak constant impedance load at bus $i$

peak constant current load at bus $i$;

peak constant power load at bus $i$

constant impedance load level relative to the peak at time $t$

constant current load level relative to the peak at time $t$

constant power load level relative to the peak at time $t$

GD generation level relative to the nominal capacity at time $t$

curtailment factor

minimum/maximum voltage limits

step of voltage variation of the device at bus $i$

minimum/maximum tap position of the device at bus $i$

rated capacity of substation transformer

maximum number of tap changes of the device at bus $i$ during the planning horizon $a_{m} / b_{m}$

$\alpha_{u}, \beta_{u}, \delta_{u}$

$v_{\mathrm{ci}} / v_{\mathrm{r}} / v_{\mathrm{co}}$

$\xi_{t}$

Variables

$P_{i}^{i c} \quad$ size of the generator at bus $i$

$P l_{i, t} / Q l_{i, t} \quad$ active/reactive load power at bus $i$ and time $t$

$\ell_{t} \quad$ system's active power losses at time $t$

$V_{i, t} \quad$ voltage magnitude at bus $i$ and time $t$

$P_{i, j, t} / Q_{i, j, t} \quad$ active/reactive power flow from bus $i$ to bus $j$ at

$P g_{i, t} / Q g_{i, t} \quad$ active/reactive power supplied at bus $i$ and time $t$

$P s_{t} / Q s_{t} \quad$ active/reactive power supplied by the substation at time $t$

$P_{i, t}^{\text {curt }} \quad$ active power curtailment from the generator at bus $i$

$P_{i, t}^{\text {av }} \quad$ available active power from the generator at bus $i$

$\operatorname{tap}_{i, t} \quad$ tap position of the device at bus $i$ and time $t$

$V_{i, t}^{\mathrm{in}} / V_{i, t}^{\text {out }} \quad$ output/input voltage of the voltage regulator at bus $i$

$W_{i, j, t}^{P} / W_{i, j, t}^{Q} \quad$ linear approximation of $P_{i, j, t}^{2} / Q_{i, j, t}^{2}$

$\chi_{i, t} / \phi_{i, t} / \theta_{i, t, u}$ dual variable corresponding to constraints (15)/ $(18) /(34)$

$F_{t}^{w t} \quad$ probabilistic distribution of $\omega_{t}$

$F_{t}^{Z} / F_{t}^{I} / F_{t}^{P} \quad$ probabilistic distribution of $\zeta_{t}^{Z} / \zeta_{t}^{I} / \zeta_{t}^{P}$

\section{Introduction}

Nowadays, there exists a trend in increasing the share of distributed generation (DG), primarily based on renewable energy sources (RES), to supply the global electric energy consumption [1]. In order to achieve RES targets, the participation of third party 
investors in developing RES-based DG projects is being promoted through the liberalisation of electricity markets. In this new scenario, planning the DG expansion is no longer possible because distribution network operators (DNOs) must provide network access to all connection requests, irrespective of the DG size and location. Moreover, when the current network's statutory or physical limits are reached, the DNOs must incur in actualisation of their assets to allow new DG connections. Thus, the energy market restructuring has led to a new paradigm related to the determining of the maximum capacity of new DG able to be connected to a distribution network without requiring network upgrade, sometimes referred to as maximum hosting capacity (MHC).

In general, the MHC problem is solved through optimisation techniques, where the objective is the maximisation of the DG installed capacity subject to network constraints, usually imposed by voltage and thermal limits. Some proposals have also sought to include voltage step constraints derived from outages of generators [2], and fault level constraints [3]. Other studies foresee the implementation of active network management (ANM) schemes, designed to dynamically manage constraints that depend on the variability of RES and load. Examples of ANM schemes are: coordinated voltage control, DG generation curtailment and reactive power compensation. In [4], a methodology based on linear programming is presented, which maximises the energy delivered from DG applying energy curtailment. AlKaabi et al. [5] proposed various ANM schemes based on the photovoltaic (PV) inverter control for reactive power compensation, applied to maximise the total wind-DG penetration. A multi-period non-linear programing formulation of the optimal power flow (OPF) is used in [6] to evaluate the maximum wind energy exploitation, applying coordinated voltage control through on-load tap changer (OLTC), energy curtailment and DG power factor control. In [4-6], the settings of the ANM schemes are determined independently for each time period. However, the controllable resources required by ANM schemes are limited in availability and must be efficiently managed during the planning horizon; therefore, temporal interdependencies need to be considered. To this end, in [7] a multi-period AC OPF-based technique is proposed for solving the MHC problem considering temporal interdependencies for limiting the amount of curtailed energy, while applying DG power factor control and coordinated voltage control via OLTC and voltage regulators (VRs). In [8], a similar technique as in [7] was proposed, but also exploring the potential benefits of network reconfiguration.

In the previous works [2-8], the variability of load and generation is taken into account by using historical data, and techniques for aggregation of time periods are applied to reduce the problem size. Recently, the effects of load and generation uncertainties in the MHC are being investigated. In [9], the applicability of robust optimisation to maximise the installed capacity of solar PV DG, coordinating the operation of an OLTC and static VAR compensators when load and generation uncertainties are included, is studied. Zio et al. [10] addressed the MHC problem with a Monte Carlo technique to include the load uncertain behaviour. In both proposals $[9,10]$, successive load flow calculations are performed to simulate each time period in the planning horizon and, therefore, temporal interdependencies are not included in the analysis.

From the literature review, it can be seen that the MHC of distribution networks has been evaluated considering: single time period analyses [2, 3], multi-period analyses [4-6], multi-period analyses with temporal interdependencies $[7,8]$ and load and/or generation uncertainties $[9,10]$. Nevertheless, any of these studies simultaneously considers multi-period analyses, temporal interdependencies and load and generation uncertainties. Moreover, in [2-10], the main concern in the determination of the $\mathrm{MHC}$ is the fulfilment of essential network constraints. However, the procurement of high DG penetration levels may greatly impact energy losses and, therefore, the trade-off between DG hosting capacity and energy losses also needs to be taken into account. Only in $[6,7]$, the effects of high DG penetration levels on energy losses are discussed, and in [4] the MHC is evaluated taking into account the energy losses by including a loss adjustment factor in the objective function. Additionally, in all of these studies, loads are modelled as constant power loads and, consequently, the relationship between DG and energy consumption of voltagedependent loads is disregarded. This relationship is an important issue that must be investigated because, as the DG penetration level increases, higher voltage levels are expected to supply the load, and this may result in an increase of energy consumption.

In this work, a multi-period and multiobjective linearised OPFbased optimisation algorithm is proposed to estimate the quantity of variable DG that can be accommodated in a medium-voltage (MV) distribution network considering the efficient energy use. Here, energy efficiency is related to the amounts of energy losses in network's lines and energy consumption of loads. The algorithm seeks to maximise the installed DG capacity and to minimise both the energy consumption of loads and energy losses, while taking account of network constraints. The effect of voltage on demand is included by modelling the loads through the ZIP load model. The coordinated operation of VRs and OLTC, and the management of DG generation curtailment and reactive power compensation from DG are used to improve network utilisation and energy efficiency. Furthermore, the effects of load and generation uncertainties are addressed through a two-stage stochastic programing formulation of the multiobjective problem, where one objective function is related to the first stage decisions and the other objective function is related to the recourse actions. A deterministic approximation to the multiobjective stochastic problem is generated using a scenario generation and reduction process. The resulting multiobjective deterministic problem is solved simultaneously applying the econstrained method [11] and a decomposition technique based on the L-shaped method to obtain a set of non-inferior solutions.

The major contributions of this work are as follows:

- The MHC of MV distribution networks is evaluated simultaneously considering the efficient energy use, load and generation uncertainties and the implementation of various ANM schemes.

- A novel multi-period, multiobjective, two-stage stochastic formulation of the MHC problem, which includes temporal interdependencies and the load-to-voltage (LTV) dependence through the ZIP load model.

- A solution strategy that integrates the e-constrained method and the L-shaped method to deal with the multiobjective stochastic formulation.

\section{MHC, ANM and energy efficiency}

Opening the energy market to the participation of independent investors in the development of DG projects demands the DNOs to efficiently manage the network utilisation; because the capacity for new connections is limited and network expansion results expensive and usually restricted by environmental and social concerns. In this sense, DNOs would be interested in determining the locations where more DG can be accommodated and incentivise the developments in that areas, for example by applying differentiated connection rates. However, when the priority is in the exploitation of RES, arises the need for more sophisticated strategies to deal with the generation variability and its lack of correlation with demand; otherwise, the DG hosting capacity would be constrained by extreme generation and demand scenarios that seldom occur. In this scenario, the concept of ANM becomes fundamental as a means for integrating large amounts of variable DG.

ANM incorporates the interaction between planning, access and connection, and operational timeframes, and was devised to coordinate the development of generation, energy storage and flexible demand [1]. Moreover, its implementation calls for the evolution of regulatory and commercial frameworks and, thereby, providing tools to promote DG connections under non-firm access agreements and market-based procurement of flexibility and ancillary services from DG. Thus, with the appropriate communication, monitoring and control infrastructure, coordinated smart control schemes, referred to as ANM schemes, can be put in 
place to optimally manage DG capabilities and other controllable network elements so that network utilisation can be maximised.

\subsection{Energy efficiency}

Some technical difficulties may impact the efficiency in electric power systems, and among them, DG is gaining relevance. It has been shown DG penetration above certain level can increase losses and power demand of voltage-dependent loads, if no corrective actions are undertaken [12-14]. In this regard, the LTV dependence has been included in studies for DG planning $[15,16]$ and operation $[17,18]$; nevertheless, to the best of the author's knowledge, the interaction between LTV dependence and MHC has not been yet studied.

When the priority is in the exploitation of RES, ANM schemes are deployed to maximise the DG hosting capacity of existing distribution networks. However, in the search for a more sustainable energy system, improving energy efficiency possesses a high priority. Therefore, ANM schemes need to be applied in way to maintain low levels of energy losses and energy consumption of loads when the DG hosting capacity is maximised.

\section{Mathematical formulation}

\subsection{DG modelling}

Variable DG interfaced with synchronous generators (SGs) and power electronics converters has the technical capability to supply more continuous reactive output than capacitor banks and, with appropriate controls, it can respond faster and with higher control resolution. These properties along with a rapid development of DG are moving the industry to allow DG to provide voltage regulation $[19,20]$. In this work, the focus is on wind power (WP) generation, for which we define the reactive power capability as in (1). This simplified formulation can be representative of a wind turbine interfaced with a SG or a full power electronics converter.

$$
\left|Q_{\max }\right|=\sqrt{\left(S_{\max }^{2}-P g^{2}\right)}
$$

In relation to the mode of operation of DG, it is considered that, when required, the active power generation can be reduced to overcome network constraints, to free reactive capacity and to regulate voltage. Additionally, it is considered during low wind speed scenarios, at zero active power generation, the DG can still provide reactive power support.

\subsection{Problem formulation}

When energy efficiency and high penetration levels of RES-based generation are pursued, DNOs are committed to optimise utilisation of their networks and to prevent unnecessary energy usage; whereas DG developers are encouraged to invest in the construction of new DG units. To achieve their objectives, DNOs can offer incentives to DG developers to site their units in appropriate locations and procure flexibility and ancillary services from DG, as long as it results more cost effective than reinforcing the network. By providing support to DNOs, DG developers have the possibility to increase their participation in the energy market, because higher DG capacities can be installed, and to receive additional benefits from the provision of reactive compensation and flexibility.

The MHC problem considering energy efficiency and load/ generation uncertainties is modelled as a mixed-integer, non-linear, multiobjective, two-stage, stochastic programming problem. Stochastic programming requires that the probability distributions of the uncertain data are known or can be estimated. This method was chosen because: (i) there are good approximations of the probability distributions governing the uncertainties of RES and load [15, 17, 21]; (ii) stochastic programming is a well-established method that has been the subject of many studies and has been applied to many power system problems with satisfactory results $[22,23]$. Below is presented the mathematical formulation of the problem:

$$
\max : f_{1}=\sum_{\substack{i \in G \\ P \\ i}}^{i c}
$$

$$
\min : f_{2}=\mathbb{E}\left[\min : \tilde{f}_{2}\left(\xi_{t}\right)=\sum_{t \in T}\left(\sum_{i \in \Omega_{b}} P l_{i, t}\left(\xi_{t}\right)+\ell_{t}\left(\xi_{t}\right)\right)\right]
$$

Subject to: $\operatorname{tap}_{i}^{\min } \leq \operatorname{tap}_{i, t} \leq \operatorname{tap}_{i}^{\max }, \quad \forall i \in R, \forall t \in T$

$$
\sum_{t=1}^{|T|-1}\left|\operatorname{tap}_{i, t+1}-\operatorname{tap}_{i, t}\right| \leq \mathrm{TAP}_{i}^{\max }, \quad \forall i \in R
$$

$\sum_{i j \in \Omega_{l}} P_{i, j, t}\left(\boldsymbol{\xi}_{t}\right)=P_{k, i, t}\left(\boldsymbol{\xi}_{t}\right)-P l_{i, t}\left(\boldsymbol{\xi}_{t}\right)+P g_{i, t}\left(\boldsymbol{\xi}_{t}\right), \quad \forall i \in \Omega_{b}, \forall t$ $\in T$

$$
\sum_{i j \in \Omega_{l}} Q_{i, j, t}\left(\boldsymbol{\xi}_{t}\right)=Q_{k, i, t}\left(\boldsymbol{\xi}_{t}\right)-Q l_{i, t}\left(\boldsymbol{\xi}_{t}\right)+Q g_{i, t}\left(\boldsymbol{\xi}_{t}\right), \quad \forall i \in \Omega_{b},
$$

$V_{j, t}\left(\boldsymbol{\xi}_{t}\right)=V_{i, t}\left(\boldsymbol{\xi}_{t}\right)-\frac{r_{i, j} P_{i, j, t}\left(\boldsymbol{\xi}_{t}\right)+x_{i, j} Q_{i, j, t}\left(\boldsymbol{\xi}_{t}\right)}{V_{1, t}}, \quad \forall i j \in \Omega_{l}, \forall t$

$\in T$

$$
\begin{aligned}
& \ell_{t}\left(\boldsymbol{\xi}_{t}\right)=\sum_{i j \in \Omega_{l}} r_{i, j} \frac{P_{i, j, t}^{2}\left(\boldsymbol{\xi}_{t}\right)+Q_{i, j, t}^{2}\left(\boldsymbol{\xi}_{t}\right)}{V_{1, t}^{2}\left(\boldsymbol{\xi}_{t}\right)}, \quad \forall t \in T \\
& P l_{i, t}\left(\boldsymbol{\xi}_{t}\right)=P l_{i}^{Z} \zeta_{t}^{Z} V_{i, t}^{2}\left(\boldsymbol{\xi}_{t}\right)+P l_{i}^{I} \zeta_{t}^{I} V_{i, t}\left(\boldsymbol{\xi}_{\xi}\right)+P l_{i}^{P} \zeta_{t}^{P}, \quad \forall i \in \Omega_{b}, \\
& \forall t \in T
\end{aligned}
$$

$Q l_{i, t}\left(\boldsymbol{\xi}_{t}\right)=Q l_{i}^{Z} \zeta_{t}^{Z} V_{i t}^{2}\left(\boldsymbol{\xi}_{t}\right)+Q l_{i}^{I} \zeta_{t}^{I} V_{i, t}\left(\boldsymbol{\xi}_{t}\right)+Q l_{i}^{P} \zeta_{t}^{P}, \quad \forall i \in \Omega_{b}$, $\forall t \in T$

$$
\begin{gathered}
P g_{i, t}\left(\boldsymbol{\xi}_{t}\right)=P_{i, t}^{\mathrm{av}}\left(\boldsymbol{\xi}_{t}\right)-P_{i, t}^{\mathrm{curt}}\left(\boldsymbol{\xi}_{t}\right), \quad \forall i \in G, \quad \forall t \in T \\
P_{i, t}^{\mathrm{curt}}\left(\boldsymbol{\xi}_{t}\right) \leq P_{i, t}^{\mathrm{av}}\left(\boldsymbol{\xi}_{t}\right), \quad \forall i \in G, \quad \forall t \in T \\
\sum_{t \in T} P_{i, t}^{\mathrm{curt}}\left(\boldsymbol{\xi}_{t}\right) \leq \gamma^{c} \sum_{t \in T} P_{i, t}^{\mathrm{av}}\left(\boldsymbol{\xi}_{t}\right), \quad \forall i \in G \\
P_{i, t}^{\mathrm{av}}\left(\boldsymbol{\xi}_{t}\right)=\omega_{t} P_{i}^{i c}: \chi_{i, t}, \quad \forall i \in G, \forall t \in T \\
P g_{1, t}\left(\boldsymbol{\xi}_{t}\right)=P s_{t}\left(\boldsymbol{\xi}_{t}\right), \quad \forall t \in T \\
Q g_{1, t}\left(\boldsymbol{\xi}_{t}\right)=Q s_{t}\left(\boldsymbol{\xi}_{t}\right), \quad \forall t \in T
\end{gathered}
$$

$V_{i, t}^{\text {out }}\left(\xi_{t}\right)=V_{i, t}^{\text {in }}\left(\boldsymbol{\xi}_{t}\right)+\Delta \operatorname{tap}_{i} \operatorname{tap}_{i, t} \quad: \phi_{i, t}, \quad \forall i \in R, \quad \forall t \in T$

$$
V_{1, t}^{\mathrm{in}}\left(\boldsymbol{\xi}_{t}\right)=1, \quad \forall t \in T
$$

$$
V_{\min } \leq V_{i, t}\left(\boldsymbol{\xi}_{t}\right) \leq V_{\max }, \quad \forall i \in \Omega_{b}, \forall t \in T
$$

$$
\begin{gathered}
\left|Q g_{i, t}\left(\boldsymbol{\xi}_{t}\right)\right| \leq \sqrt{\left(P_{i}^{i c}\right)^{2}-P g_{i, t}^{2}\left(\boldsymbol{\xi}_{t}\right)}, \quad \forall i \in G, \quad \forall t \in T \\
P s_{t}^{2}\left(\boldsymbol{\xi}_{t}\right)+Q s_{t}^{2}\left(\boldsymbol{\xi}_{t}\right) \leq S_{1, \max }^{2}, \quad \forall t \in T \\
\boldsymbol{\xi}_{t}=\left(\omega_{t}, \zeta_{t}^{Z}, \zeta_{t}^{I}, \zeta_{t}^{P}\right), \quad \forall t \in T \\
\omega_{t} \in F_{t}^{\mathrm{wt}}, \quad \forall t \in T
\end{gathered}
$$

$$
\zeta_{t}^{Z} \in F_{t}^{Z}, \zeta_{t}^{I} \in F_{t}^{I}, \zeta_{t}^{P} \in F_{t}^{P}, \quad \forall t \in T
$$


In the above formulation, the first stage problem is a multiobjective problem given by the expressions in (2)-(5). The expression in (2) corresponds to the maximisation of the total installed DG capacity in a set of predefined network buses $G$. The expression in (3) corresponds to the minimisation of the expectation of the second stage objective $\tilde{f}_{2}\left(\boldsymbol{\xi}_{t}\right)$ taken over all possible realisations of the uncertain data $\xi_{t}$. Constraint (4) defines the maximum and minimum tap positions of VRs and OLTCs located at the buses $i$ in the set $R$. Constraint (5) limits the number of switching operations of VRs and OLTCs during the planning horizon. Therefore, the first stage problem determines the tap position of VRs and OLTCs $\operatorname{tap}_{i, t}$ and the size of generators $P_{i}^{i c}$.

For each realisation of $\xi_{t}$, the second stage problem minimises the total energy consumption (energy consumption of loads plus energy losses in lines) represented by $\tilde{f}_{2}\left(\boldsymbol{\xi}_{t}\right)$, subject to constraints (6)-(25). The decision variables in the second stage problem are the generation curtailment $P_{i, t}^{\text {curt }}\left(\boldsymbol{\xi}_{t}\right)$ and the reactive power injected from the DG $Q g_{i, t}\left(\boldsymbol{\xi}_{t}\right)$.

Constraints (6)-(8) are the linearised DistFlow equations for nodal power flows representation. These equations were initially described in [24], and have been extensively used in the literature $[9,15,17]$. Equation (9) describes the active power losses in network branches for each time period. In (10) and (11), the ZIP model is used to represent the voltage-dependent behaviour of loads for active and reactive power. In the ZIP model, loads consist of constant impedance $(Z)$, constant current $(I)$ and constant power $(P)$ components, and the degree of dependence with voltage varies according to the percentage of participation of each component [25]. In this work, the participation of each component at each bus and time period is calculated as the product between the peak load and the load level relative to peak, which in this case is a random parameter.

In (12), the actual active power injected by a generator at a specific bus and time period is calculated. Constraint (13) establishes the generation curtailment cannot be greater than the available active power. In (14), the amount of curtailed energy from each generator during the planning horizon is restricted to a maximum percentage of the energy that can be generated. In (15), the available active power of a generator depends on the installed capacity and on the generation level relative to that capacity $\omega_{t}$, which is a random parameter dependent on the wind speed. Constraints (16) and (17) correspond to the active and reactive power injections at the substation. Constraint (18) models the operation of VRs and OLTCs. Constraint (19) assumes the voltage at the primary side of the substation transformer is kept to a constant value of 1.0 p.u. Constraint (20) indicates the voltage at each node should be within the statutory range. Constraint (21) defines the reactive power capability of generators as discussed in the previous section. Here, the rated capacity of a generator is given by $P_{i}^{i c}$. Constraint (22) limits the apparent power flowing through the substation transformer. Equation (23) defines the random vector $\xi_{t}$. Expressions (24) and (25) assume that the generation levels and the demand levels are random parameters that can be represented using probability density functions (PDF), as will be discussed later.

Non-linearities in the above model are reduced. Constraint (5) is linearised by transforming it into the equivalent form shown in the following equations:

$$
\begin{gathered}
\lambda_{i, t} \geq \operatorname{tap}_{i, t+1}-\operatorname{tap}_{i, t}, \quad \forall i \in R, \forall t \in T \\
\lambda_{i, t} \geq \operatorname{tap}_{i, t}-\operatorname{tap}_{i, t+1}, \quad \forall i \in R, \forall t \in T \\
\sum_{t \in T} \lambda_{i, t} \leq \operatorname{TAP}_{i}^{\max }, \quad \forall i \in R
\end{gathered}
$$

Constraint (9) is linearised by replacing $P_{i, j, t}^{2}$ and $Q_{i, j, t}^{2}$ with piecewise linear approximations, and assuming the secondary voltage of the transformer at the substation, $V_{1, t}^{2}$, is equal to 1 [24].
Thus, (9) can be written as (29), and constraints (30) and (31) are added

$$
\begin{gathered}
\ell_{t}\left(\boldsymbol{\xi}_{t}\right)=\sum_{i j \in \Omega_{l}} r_{i j}\left(W_{i, j, t}^{P}\left(\xi_{t}\right)+W_{i, j, t}^{Q}\left(\boldsymbol{\xi}_{t}\right)\right), \quad \forall t \in T \\
W_{i, j, t}^{P}\left(\boldsymbol{\xi}_{t}\right) \geq a_{m}+b_{m} P_{i, j, t}\left(\boldsymbol{\xi}_{t}\right), \quad \forall i j \in \Omega_{l}, \quad \forall t \in T, \quad \forall m \in M \\
W_{i, j, t}^{Q}\left(\boldsymbol{\xi}_{t}\right) \geq a_{m}+b_{m} Q_{i, j, t}\left(\boldsymbol{\xi}_{t}\right), \quad \forall i j \in \Omega_{l}, \quad \forall t \in T, \forall m \in M
\end{gathered}
$$

The quality of the above approximation increases with the number of linear segments approximating the quadratic terms; however, this number must be carefully chosen to avoid excessive use of computational resources in solving the problem. The quadratic expression in constraints (10) and (11) is linearised by using Taylor's expansion around $V_{i, t}=1$ and ignoring the second- and high-order terms as shown in (32) and (33). Note that, the error introduced by this linearisation is small since, according to (20), $V_{i, t}$ at any time and bus is close to 1

$$
\begin{aligned}
& P l_{i, t}\left(\boldsymbol{\xi}_{t}\right)=P l_{i}^{Z} \zeta_{t}^{Z}\left(2 V_{i, t}\left(\boldsymbol{\xi}_{t}\right)-1\right)+P l_{i}^{I} \zeta_{t}^{I} V_{i, t}\left(\xi_{t}\right)+P l_{i}^{P} \zeta_{t}^{P}, \quad \forall i \\
& \in \Omega_{b}, \quad \forall t \in T \\
& Q l_{i, t}\left(\boldsymbol{\xi}_{t}\right)=Q l_{i}^{Z} \zeta_{t}^{Z}\left(2 V_{i, t}\left(\boldsymbol{\xi}_{t}\right)-1\right)+Q l_{i}^{I} \zeta_{t}^{I} V_{i, t}\left(\boldsymbol{\xi}_{t}\right)+Q l_{i}^{P} \zeta_{t}^{P}, \quad \forall i \\
& \in \Omega_{b}, \forall t \in T
\end{aligned}
$$

Constraints (21) and (22) are also linearised through piecewise linear approximations as shown in (34) and (35). Here, the method described in [9] is used to calculate the linear segments

$$
\begin{aligned}
& \alpha_{u} P g_{i, t}\left(\boldsymbol{\xi}_{t}\right)+\beta_{u} Q g_{i, t}\left(\boldsymbol{\xi}_{t}\right)+\delta_{u} P_{i}^{i c} \leq 0: \theta_{i, t, u}, \quad \forall i \in G, \quad \forall t \in T, \\
& \forall u \in U \\
& \quad \alpha_{u} P s_{t}\left(\boldsymbol{\xi}_{t}\right)+\beta_{u} Q s_{t}\left(\boldsymbol{\xi}_{t}\right)+\delta_{u} S_{\max } \leq 0, \quad \forall t \in T, \quad \forall u \in U
\end{aligned}
$$

\section{Uncertainty characterisation}

In the model presented in the previous section, uncertainties are related to errors in predicting load levels and wind speed. In this work, wind forecast errors are modelled by a special case of the Weibull PDF called Rayleigh PDF [21], expressed as follows:

$$
f_{R}\left(v_{t}\right)=\left(\frac{2 v_{t}}{c_{t}^{2}}\right) \exp \left[-\left(\frac{v_{t}}{c_{t}}\right)^{2}\right], \quad \forall t \in T
$$

The scale parameter $c_{t}$ is calculated as in (37), where $v_{\mu, t}$ is the mean value of the PDF, which, in this work, is the predicted wind speed for a specific time period. Therefore, (36) models, for each time period $t$, the occurrence of wind speed values $v_{t}$ when a certain prediction value $v_{\mu, t}$ has been forecasted

$$
c_{t}=\frac{2}{\sqrt{\pi}} v_{\mu, t}, \quad \forall t \in T
$$

For a realisation of $v_{t}$ the conversion to WP is determined by the power performance curve of the wind turbine. A piecewise linear approximation to this curve is expressed in (38). Note that for purposes of simplicity, it is assumed all DG locations are subject to the same wind speed

$$
\omega_{t}=\left\{\begin{array}{ll}
0, & 0 \leq v_{t}<v_{\mathrm{ci}} \\
\frac{v_{t}-v_{\mathrm{ci}}}{v_{\mathrm{r}}-v_{\mathrm{ci}}} & v_{\mathrm{ci}} \leq v_{t}<v_{\mathrm{r}} \\
1, & v_{\mathrm{r}} \leq v_{t}<v_{\mathrm{co}} \\
0, & v_{\mathrm{co}}<v_{t}
\end{array} \quad \forall t \in T\right.
$$

As for the load forecast errors, they are assumed to be normally distributed around the predicted values with standard deviation 
equal to $2 \%$ of the expected demand $[15,17]$. Therefore, for each time period $t$, the random vector $\boldsymbol{\xi}_{t}$ is formed by one Rayleigh PDF and three normal PDFs, describing the wind speed and load forecast errors, respectively.

\section{Solution methodology}

\subsection{Sampling and scenario reduction}

An approach for solving two-stage stochastic problems is through Monte Carlo simulations. The accuracy of Monte Carlo methods increases with the size of the generated random sample; however, this also implies increasing the computational complexity. Consequently, it becomes necessary a means for estimating a reduced number of scenarios that efficiently approximate the system uncertainty. To that end, in this work it is proposed the implementation of the simultaneous backward reduction (SBR) technique $[17,26]$. Assuming there are $N$ initial scenarios, denoted by $\tau_{s}(s \in S:=\{1, \ldots, N\})$, each one with probability $\rho_{s}$, SBR is to reduce them into $n$ scenarios, denoted by $\tau_{s}^{\prime}(s \in S:=\{1, \ldots, n\})$, each one with probability $\rho_{s}^{\prime}$. The sampling and scenario reduction procedure allows to write the stochastic formulation in Section 3.2 as a mixed-integer multiobjective deterministic (MIMD) optimisation problem, defined over a reduced number of scenarios $n$

$$
\begin{gathered}
\max : f_{1}=\sum_{i \in G} P_{i}^{i c} \\
\min : f_{2}=\sum_{s \in S} \rho_{s}^{\prime} \sum_{t \in T}\left(\sum_{i \in \Omega_{b}} P l_{i, t, s}+\ell_{t, s}\right)
\end{gathered}
$$

Subject to: (4), (26) - (28)(6) - (8), (12) - (20), (29) - (35), $\forall s \in S$

\subsection{Multibojective optimisation}

In a multiobjective problem, the degree of conflict, or tradeoff, among objectives can be estimated through the set of non-inferior or Pareto optimal solutions. In this work, the e-constrained method [11] is used to generate a representative subset of the non-inferior set for objectives $f_{1}$ and $f_{2}$. In the e-constrained method one objective is selected to be optimised, with the other objective functions regarded as constraints. Here, the MIMD problem defined in the previous subsection is solved by optimising $f_{1}$ with $f_{2}$ included in the constraint set, expressed as follows:

$$
\tilde{f}_{2, s}=\sum_{t \in T}\left(\sum_{\substack{i \in \Omega_{b} \\ P}} l_{i, t, s}+\ell_{t, s}\right) \leq \varepsilon_{s}^{d}, \quad \forall s \in S
$$

where $\tilde{f}_{2, s}$ corresponds to the objective function of the second stage problem evaluated for each scenario $s \in S$. Note that, instead of a single constraint, $f_{2}$ is split into $n$ constraints, one for each scenario $s$, to keep the separable structure of the problem and apply the Lshaped method for its solution. Each parameter $\varepsilon_{s}^{d}, s \in S$, is within a range of values limited below by the optimal value $\varepsilon_{s}^{\text {min }}$ of the objective $\tilde{f}_{2, s}$, obtained when the MIMD problem is solved optimising only $f_{2}$, and above by the worst of the values $\varepsilon_{S}^{\max }$ of the objective $\tilde{f}_{2, s}$, obtained when the MIMD problem is solved optimising only $f_{1}$. By ranging $\varepsilon_{s}^{d}, s \in S$, in the transformed problem, with $f_{2}$ considered as a constraint, and resolving each new problem the non-inferior set is generated. For each scenario $s \in S$, the values of the parameters $\varepsilon_{s}^{d}$ are determined using the following formula:

$$
\varepsilon_{s}^{d}=\varepsilon_{s}^{\min }+\left[\frac{d-1}{h-1}\right]\left(\varepsilon_{s}^{\max }-\varepsilon_{s}^{\min }\right), \quad \forall d \in\{1, \ldots, h\}
$$

where $h$ is the number of non-inferior solutions to be generated. When the e-constrained method is applied to find the non-inferior set of the MIMD problem, three different problems need to be solved: the problem that optimises $f_{1}, \mathrm{OP}^{1}$; the problem that optimises $f_{2}, \mathrm{OP}^{2}$; and the problem that optimises $f_{1}$ with $f_{2}$ regarded as a constraint, $\mathrm{OP}^{3}$. The procedure for the e-constrained method can be summarised as follows:

Step 1: Solve $\mathrm{OP}^{1}$ and $\mathrm{OP}^{2}$;

Step 2: Determine the values of $\varepsilon_{s}^{d}, s \in S$, using (42);

Step 3: For each $d \in\{2, \ldots, h-1\}$, solve $\mathrm{OP}^{3}$.

\subsection{L-shaped method}

A multi-cut version of the L-shaped method $[27,28]$ is used to solve the three problems defined in the previous subsection. Using this method each problem can be decomposed into a mixed-integer linear master problem, and $n$ pure linear sub-problems, one corresponding to each scenario $s$. However, in order to reduce the search space, in this work, a partial decomposition technique is applied [29]. That is, a subset of sub-problems $\bar{S} \subseteq S$ is retained in the master problem to provide a tighter formulation. Here, the retained sub-problems are arbitrarily selected. For each iteration $j$, the solution of the master problem provides the tap positions of the OLTC and VRs $\operatorname{tap}_{i, t}^{(j)}$, the size of the generators $P_{i}^{i c(j)}$, and the solution vector of the retained sub-problems. With the variables $P_{i}^{i c}$ and $\operatorname{tap}_{i, t}$ fixed at the values found in the master problem, the subproblems $s \in S \backslash \bar{S}$ are solved and the values of their dual variables $\chi_{i, t, s}^{(j)}, \phi_{i, t, s}^{(j)}$ and $\theta_{i, t, u, s}^{(j)}$, and objective functions $\tilde{f}_{2, s}^{(j)}$ are used to derive constraints on the master problem. For $\mathrm{OP}^{1}$, the sub-problems are given by the deterministic representation of constraints (6)-(8), (12)-(20) and (29)-(35), taking one scenario at a time. For $\mathrm{OP}^{2}$ the sub-problems optimise $\tilde{f}_{2, s}$ subject to the same constraints as in $\mathrm{OP}^{1}$. Sub-problems of $\mathrm{OP}^{3}$ are the same as in $\mathrm{OP}^{1}$ but including constraint (41). The general formulation of the master problem at iteration $j$ for $\mathrm{OP}^{1}, \mathrm{OP}^{2}, \mathrm{OP}^{3}$ is as follows:

$$
\min :-f_{1}+\sum_{s \in \bar{S}} \rho_{s}^{\prime} \sum_{t \in T}\left(\sum_{i \in \Omega_{b}} P l_{i, t, s}+\ell_{t, s}\right)+\sum_{s \in S, \bar{S}} \rho_{s}^{\prime} Z_{s}
$$

Subject to:

$$
\text { (4), (26) - (28) }
$$

$$
\begin{gathered}
\text { (6) - (8), (12)- (20), (29)- (35), } \quad \forall s \in \bar{S} \\
\Gamma^{(l)} Z_{s}-\tilde{f}_{2, s}^{(l)} \geq-\sum_{i \in G} \sum_{t \in T} \chi_{i, t, s}^{(l)} \omega_{t, s}\left(P_{i}^{i c(l)}-P_{i}^{i c}\right)- \\
\sum_{i \in R} \sum_{t \in T} \phi_{i, t, s}^{(l)} \Delta \operatorname{tap}_{i}\left(\operatorname{tap}_{i, t}^{(l)}-\operatorname{tap}_{i, t}\right)+ \\
\sum_{i \in G} \sum_{t \in T} \sum_{u \in U} \theta_{i, t, u, s}^{(l)} \delta_{u}\left(P_{i}^{i c(l)}-P_{i}^{i c}\right) \\
\forall l \in\{1, \ldots, j\}, \quad \forall s \in D^{(l)}
\end{gathered}
$$

In the above formulation, variables $Z_{s}$ replace in the master problem the objective functions of the sub-problems $s \in S \backslash \bar{S}$, and the terms $\sum_{t \in T}\left(\sum_{i \in \Omega_{b}} P l_{i, t, s}+\ell_{t, s}\right)$ are the objective functions of the retained sub-problems $\bar{S} \subseteq S$. For $\mathrm{OP}^{3}$, the retained subproblems also include constraints of the same type as (41). If $\Gamma^{(l)}=1$, constraint (44) corresponds to optimality cuts, and if $\Gamma^{(l)}=0$ constraint $(44)$ correspond to feasibility cuts. When $\mathrm{OP}^{1}$ and $\mathrm{OP}^{3}$ are solved, only feasibility cuts are generated, since the sub-problems do not have objective function; hence, only the term $-f_{1}$ in (43) is considered and, for both problems, the algorithm 


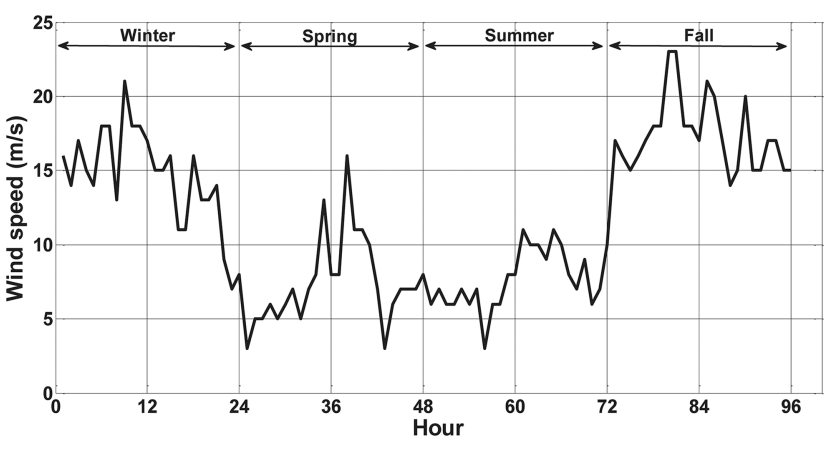

Fig. 1 Predicted wind speeds

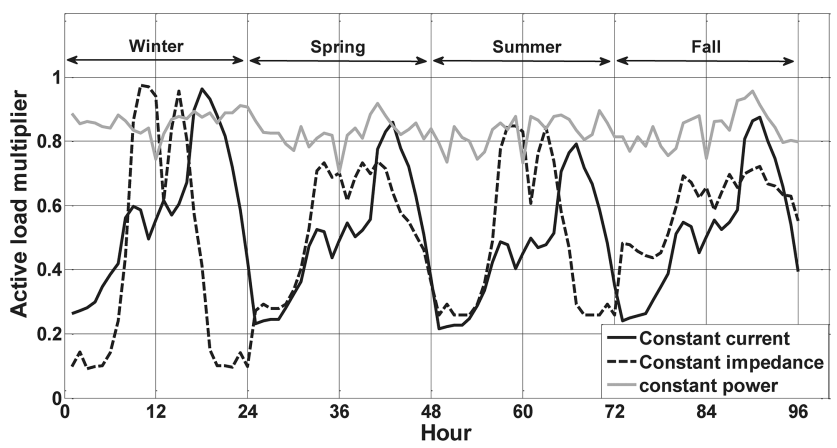

Fig. 2 Predicted load levels relative to peak load

Table 1 Load composition of the IEEE 34-bus feeder

\begin{tabular}{lcc}
\hline Load type & Participation, \% & Power factor \\
\hline$Z$ & 39.85 & 0.90 \\
$I$ & 40.70 & 0.95 \\
$P$ & 19.45 & 0.98 \\
\hline
\end{tabular}

converges when feasibility is achieved in all sub-problems. When $\mathrm{OP}^{2}$ is solved, the term $-f_{1}$ in (43) is not considered and both feasibility and optimality cuts can be generated. In this case, algorithm converges when, at iteration $j,|\mathrm{UB}-\mathrm{LB}| \leq \epsilon$, where $\mathrm{UB}$ and $\mathrm{LB}$ are, respectively, the upper and the lower bound of the optimal solution of $\mathrm{OP}^{2}$, and $\epsilon$ is the tolerance. The procedure for the L-shaped method is summarised below.

1. Initialisation: Create a set $\bar{S} \subseteq S$, let $\epsilon>0$ be given. Set an upper bound $\mathrm{UB}=\infty$, lower bound $\mathrm{LB}=-\infty$ and iteration $j=0$.

2. Solve the master problem: Obtain $\operatorname{tap}_{i, t}^{(j)}$ and $P_{i}^{i c(j)}$. If the problem being solved is $\mathrm{OP}^{2}$, also obtain $Z_{s}$ and calculate the lower bound $\mathrm{LB}=\sum_{s \in S, \bar{S}} \rho_{s}^{\prime} Z_{s}^{(j)}$. In this case, as long as no optimality cuts have been generated, the values of $Z_{s}$ are set to be zero, otherwise, the master problem would be unbounded.

3. Solve the sub-problems: If at least one sub-problem $s \in S \backslash \bar{S}$ is infeasible for the proposals of the master problem, make $\Gamma^{(j)}=0, D^{(j)}=D^{\prime(j)}$, being $D^{\prime(j)} \subseteq S \backslash \bar{S}$ the set of infeasible subproblems at iteration $j$. For the unfeasible sub-problems, the values $\chi_{i, t, s}^{(j)}, \phi_{i, t, s}^{(j)}, \theta_{i, t, u, s}^{(j)}$ and $\tilde{f}_{2, s}^{(j)}$ obtained from the phase I of the simplex method are used to form feasibility cuts in the master problem. Increase the iteration number $j=j+1$ and return to step 2. If all the sub-problems $s \in S \backslash \bar{S}$ are feasible go to step 4 .

4. Calculate upper bound: If the problem being solved is $\mathrm{OP}^{1}$ or $\mathrm{OP}^{3}$, the current solution is the final solution. If the problem being solved is $\mathrm{OP}^{2}$, calculate the upper bound $\mathrm{UB}=\min \left(\mathrm{UB}, \sum_{s \in S} \bar{s}_{s}^{\prime} \tilde{f}_{2, s}^{(j)}\right)$ and go to step 5.

5. Termination criterion: If $|\mathrm{UB}-\mathrm{LB}| \leq \epsilon$ stop the algorithm, the current solution is the final solution. Otherwise, make $\Gamma^{(j)}=1$,

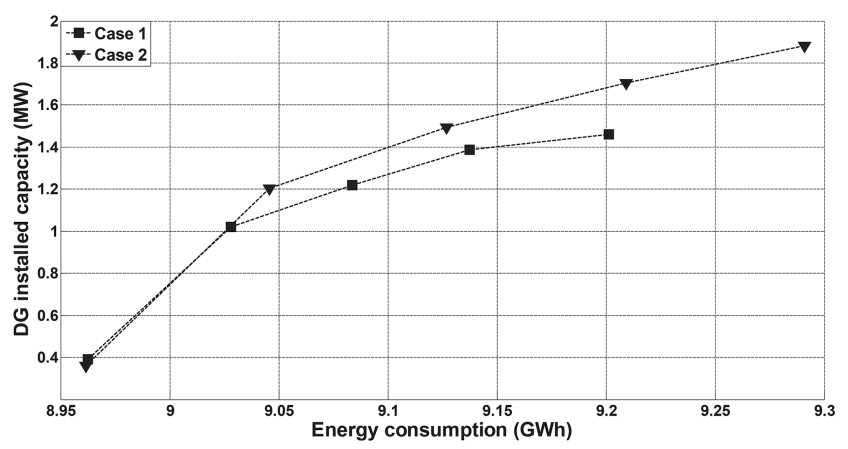

Fig. 3 Set of non-inferior solutions for the IEEE 34-bus feeder

$D^{(j)}=S \backslash \bar{S}$ and add optimality cuts to the master problem with the values $\chi_{i, t, s}^{(j)}, \phi_{i, t, s}^{(j)}, \theta_{i, t, u, s}^{(j)}$ and $\tilde{f}_{2, s}^{(j)}$ obtained from the optimal solution of the sub-problems. Increase the iteration number $j=j+1$ and return to step 2 .

\section{Case study}

The method was implemented in AMPL 20160614 calling CPLEX 12.6.3 [30]. To investigate the interaction between DG installed capacity and energy efficiency in systems with different voltage drop characteristics, tests were performed on single-phase equivalents of the IEEE 34-bus and IEEE 123-bus test feeders [31].

\subsection{Scenarios generation}

In this section, the procedure for constructing the load and generation scenarios for both feeders under study is described. In order to take into account the hourly and seasonal variations in demand and wind, each season of a 1-year planning horizon is represented by a single day divided into 24 time periods. By selecting a set of representative days it is possible to evaluate the MHC; however, in order to capture the effects of integrating large volumes of wind generation on energy efficiency, the annual energy losses and energy consumption of load should be calculated. For each representative day, predictions of load and wind profiles must be performed in a way that they approximate the general behaviour of load and wind in the days of the corresponding season. The application of sophisticated methods to generate predictions of load and wind profiles is beyond the scope of this work; here, the values shown in Figs. 1 and 2, obtained using historical average values, are used. For each profile, 200 scenarios $(N=200)$ are generated using Monte Carlo simulation to represent the prediction errors of wind speeds and load levels. Then the SBR technique is applied to reduce the number of scenarios to $15(n=15)$. For the resulting scenarios, the WP of generators is calculated using (38) with $v_{\mathrm{ci}}=5 \mathrm{~m} / \mathrm{s}, v_{\mathrm{r}}=15 \mathrm{~m} / \mathrm{s}$ and $v_{\mathrm{co}}=5 \mathrm{~m} / \mathrm{s}$. With regards to the solution procedure, the partial decomposition technique is applied retaining in the master problem only one subproblem.

\subsection{IEEE 34-bus feeder}

This feeder is characterised by including long distribution lines, nominal voltage of $24.9 \mathrm{kV}$ and peak active load of $1.769 \mathrm{MW}$. The topology and the data of this system can be found in [31]. For the tests performed here, some modifications were made. The two VRs at buses 814 and 852 , are modelled to regulate $\pm 10 \%$ of the input voltage, with $\Delta \operatorname{tap}_{i}=0.00625 \mathrm{p} . \mathrm{u} ., \operatorname{tap}_{i}^{\min }=-16$ and $\operatorname{tap}_{i}^{\max }=+16$. An OLTC is installed at the substation, capable of regulating $\pm 5 \%$ in steps of $\Delta \operatorname{tap}_{1}=0.0125 \mathrm{p}$. u . with $\operatorname{tap}_{1}^{\min }=-4$ and $\operatorname{tap}_{1}^{\max }=+4$. The feeder is supplied by a $2.5 \mathrm{MVA}$ transformer interfacing the transmission and distribution networks. Voltage limits are set to $V_{\min }=0.95 \mathrm{p} . \mathrm{u}$. and $V_{\max }=1.05 \mathrm{p} . \mathrm{u}$. Loads at buses are composed of a mix of constant impedance, constant current and constant power components; each one 
Table 2 Percentage change of total energy consumption $(\Delta E)$ and DG penetration level (PL) for the IEEE 34-bus feeder

\begin{tabular}{|c|c|c|c|c|c|c|}
\hline \multirow[t]{2}{*}{ Solution } & \multicolumn{2}{|c|}{ Case 1} & \multicolumn{2}{|c|}{ Case 2} & \multicolumn{2}{|c|}{ Case 3} \\
\hline & PL, \% & $\Delta E, \%$ & PL, \% & $\Delta E, \%$ & PL, \% & $\Delta E, \%$ \\
\hline 1 & 22.05 & -4.20 & 20.41 & -4.21 & 43.53 & -6.24 \\
\hline 2 & 57.68 & -3.50 & 68.10 & -3.31 & 147.02 & 1.91 \\
\hline 3 & 68.87 & -2.90 & 84.40 & -2.44 & 186.55 & 10.03 \\
\hline 4 & 78.34 & -2.33 & 96.42 & -1.56 & 208.44 & 18.16 \\
\hline 5 & 82.49 & -1.65 & 106.38 & -0.69 & 211.98 & 26.29 \\
\hline
\end{tabular}

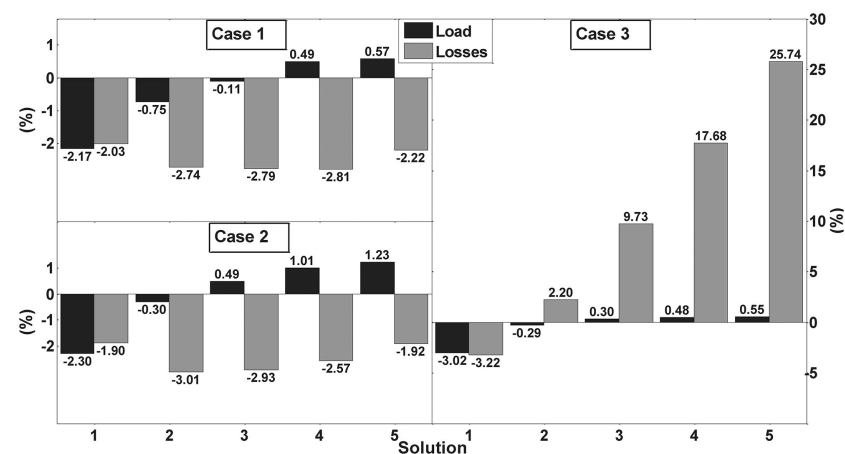

Fig. 4 Breakdown of the percentage change of energy consumption in the IEEE 34-bus feeder

assigned with a peak value. The power factor and participation at the network level of each load type are shown in Table 1.

It is also assumed that loads have constant power factor; hence the reactive load power at each bus is determined by the active load power and its power factor. The maximum number of switching operations for each VR and the OLT is set to $32\left(\mathrm{TAP}_{i}^{\max }=32\right)$; this number corresponds to the $96 \mathrm{~h}$ representing the planning horizon. Buses 838, 848, 856 and 890 were selected as candidate sites for DG allocation.

For comparison purposes, a base case is defined without DG and with the settings of the OLTC and VRs determined so as to maintain the system operating between the required limits under all load scenarios. The results of the base case for total energy consumption, energy consumption of loads and energy losses are $9.355,8.671$ and $0.684 \mathrm{GWh}$, respectively. These values correspond to the 1-year planning horizon; hence they are calculated multiplying the results obtained for the set of representative days by the number of times the size of this set is contained into 1 year. Furthermore, they represent the expected values over all scenarios.

The relationship between the amount of DG connected to the selected buses and energy consumption in the system is analysed considering the progressive implementation of ANM schemes. The following cases are studied:

- Case 1: Only coordinated voltage control.

- Case 2: Coordinated voltage control and generation curtailment limited to $\gamma^{\max }=7 \%$

- Case 3: The ANM schemes in case 2 and reactive power compensation from the DG.

For each case, five non-inferior or Pareto optimal solutions are found. The Pareto front for case 1 and case 2 are shown in Fig. 3.

The same solutions for the three cases are shown in Table 2 , where, to facilitate the analysis, the values of the first objective function are expressed as percentage changes of the total energy consumption taking the results of the base case as reference, and the values of the second objective function are expressed as DG penetration levels. In Table 2 , solution 1 corresponds to the solution that minimises the total energy consumption and solution 5 to the solution that maximises the DG capacity. Observe that, the DG penetration level is the DG installed capacity expressed as a percentage of the total system peak load. In Fig. 4, the contribution of load and losses in the percentage change of total energy consumption is shown.

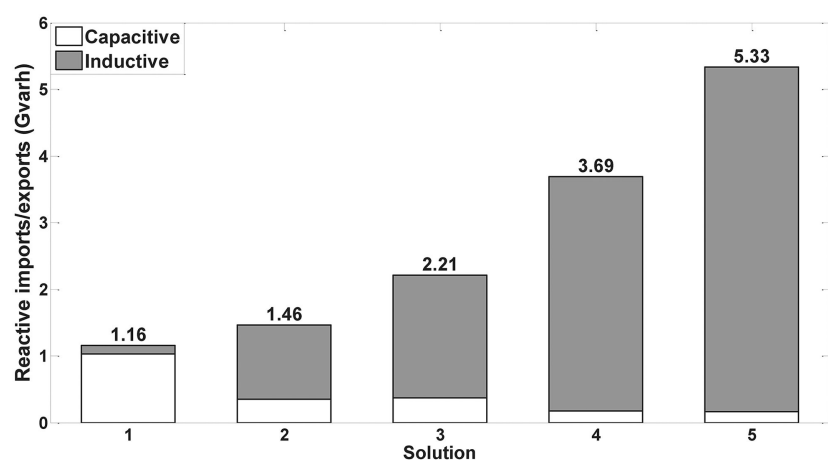

Fig. 5 Reactive power exchanges obtained for the IEEE 34-bus feeder

When the energy consumption is minimised (solution 1), the results for case 1 and case 2 are similar, since, for the obtained DG penetration levels, there is no need to perform generation curtailment to further reduce the voltage levels. In both cases, the resulting DG penetration levels are those that produce the best combination of reduced energy losses and energy consumption as shown in Fig. 4. Note that, losses are reduced when loads are locally supplied by the DG because there is a reduction in the power flows from the substation. In case 3 , the DG installed capacity is about twice as obtained in case 1 and case 2 , this results in lower energy losses and even lower energy consumption of loads.

In regard to the maximisation of the DG capacity (solution 5), the penetration level increases with the progressive implementation of more ANM schemes. In case 1, the maximum DG penetration is dependent on the capability of the OLTC and VRs to maintain adequate voltage levels; considering that their settings are determined in advance and must hold for all generation and demand scenarios. It is worth noting that, in this analysis the connection of DG is constrained only by voltage limits and the rated capacity of the substation transformer. In case 2 , generation curtailment can be performed to bring down voltage raise produced by extreme scenarios of power generation and demand. As a result, a maximum generation curtailment of $7 \%$ translates to a DG capacity $24 \%$ greater than in case 1 . In both case 1 and case 2 , the energy consumed by loads is greater than in the base case; however, the energy losses are lower because most of the energy production is locally consumed in the distribution network. As a whole, the total energy consumption is still lower than in the base case. Case 3 presents the best results in terms of allocated DG capacity, nevertheless at the cost of low energy efficiency. In this case, the DG reactive power capability is exploited to provide a finer voltage regulation than the coordinated voltage control, and less restricted than the generation curtailment. Since the generation capacity is significantly higher than the peak load, active power is exported to the transmission system. In this case, the limiting factor for allocating more DG is the capacity of the substation transformer.

When the objective is minimising the energy consumption, the DG is considered as an additional control, and its penetration level is calculated to provide the best trade-off between reduced losses and load. However, in some situation, the DNO could be interested in exploring less aggressive solutions that still provide acceptable results in terms of energy efficiency but with larger amounts of connected DG. For example, in case 1 and case 2 , if solution 2 is chosen over solution 1 , the DG penetration is tripled and the total 
Table 3 Load composition of the IEEE 123-bus feeder

\begin{tabular}{lcc}
\hline Load type & Participation, \% & Power factor \\
\hline$Z$ & 24.36 & 0.90 \\
I & 55.44 & 0.95 \\
P & 20.20 & 0.98 \\
\hline
\end{tabular}

Table 4 Percentage change of total energy consumption $(\Delta E)$ and DG penetration level (PL) for the IEEE 123-bus feeder

\begin{tabular}{lccccccc}
\hline Solution & \multicolumn{2}{c}{ Case 1 } & \multicolumn{2}{c}{ Case 2 } & \multicolumn{2}{c}{ Case 3 } \\
& $\mathrm{PL}, \%$ & $\Delta E, \%$ & $\mathrm{PL}, \%$ & $\Delta E, \%$ & $\mathrm{PL}, \%$ & $\Delta E, \%$ \\
\hline 1 & 25.21 & -4.15 & 29.80 & -4.19 & 48.71 & -4.49 \\
2 & 123.87 & -2.90 & 158.21 & -1.90 & 195.74 & -1.79 \\
3 & 152.23 & -1.80 & 205.23 & 0.18 & 224.46 & 0.65 \\
4 & 173.44 & -0.61 & 221.52 & 2.51 & 224.81 & 3.60 \\
5 & 173.44 & 0.82 & 223.48 & 4.72 & 227.39 & 6.31 \\
\hline
\end{tabular}

energy consumption is increased by $<1 \%$. This behaviour is also present in case 3 and similar results can be found if additional noninferior solutions are calculated.

When the problem is formulated to optimise only the DG installed capacity, the effects on energy efficiency are completely disregarded; thus, losses and demand may be unnecessarily large. In this sense, important benefits could be obtained if a more flexible solution is selected. This situation is more evident in case 3 , where the maximum DG capacity is achieved with energy consumption $26 \%$ above the base case. However, if solution 4 is chosen, the energy consumption is reduced $8 \%$ reducing the DG installed capacity by $<4 \%$. In case 1 and case 2 , the same pattern is observed at a lower scale but still significant.

The amounts of annual reactive power hours (varh) imported/ exported by the distribution network for the five solutions of case 3 are shown in Fig. 5, where the total of each bar corresponds to the sum of the capacitive and inductive requirements.

In this work, the assumption that capacitive sources export or generate reactive power and inductive sources import or consume reactive power is adopted. For solution 1, the network behaviour is predominantly capacitive; because the generators inject reactive power to compensate for voltage drops in scenarios of low active power generation. As the DG penetration is increased, the network becomes more inductive; because the generators consume reactive power to compensate for the voltage raise produced by the reversed active power flows. Depending on the regulations, one of these solutions can be chosen to meet restrictions on the reactive power exchanges with the transmission system while maximising the DG installed capacity.

\subsection{IEEE 123-bus feeder}

This feeder operates at a nominal voltage of $4.16 \mathrm{kV}$ and has a peak active load of $3.49 \mathrm{MW}$. Compared with the IEEE 34-bus feeder, it presents less voltage drop problems, and thus the DG capacity is expected to be less voltage constrained. The topology and the data of this system can be found in [31]. An OLTC is installed at bus 149 as well as two VRs at buses 18 and 160. The characteristics of the voltage regulation devices, their operational limits and the network's voltage limits are the same used in the IEEE 34-bus feeder. The substation transformer has nominal capacity of $5 \mathrm{MVA}$ and the candidate buses for DG allocation are $29,52,82$ and 105 . The power factor and participation of the constant impedance, constant current and constant power load components are shown in Table 3.

The results of the base case for total energy consumption, energy consumption of loads and energy losses are 17.906, 17.458 and $0.448 \mathrm{GWh}$, respectively. The same cases as in the previous section are studied. The percentage changes of the total energy consumption and the DG penetration levels for the three cases are shown in Table 4.

In this system, the DG penetration levels are higher than in the IEEE 34-bus feeder. For the three cases, the factor limiting the MHC is the rated capacity of the substation transformer. This behaviour restricts the effect of reactive power compensation to increase the DG capacity as can be seen when comparing solution 5 of case 2 and case 3 . Moreover, for the three cases, solution 5 does not present a significant improvement in the DG penetration level over solution 4, however, the energy efficiency is considerably lower.

\section{Conclusions}

The proposed method provides a comprehensive picture of the relationship between DG hosting capacity and energy efficiency, and could be used as support in the process of defining the expansion planning of DG. From the results, it was observed that, as the DG penetration approaches the maximum capacity of the network, connecting new DG becomes more expensive in terms of energy efficiency. For example, in most of the studied cases, when compared to the adjacent non-inferior solution, the solution with maximum installed DG capacity improves the DG penetration level by $<4 \%$ increasing the energy consumption more than $1 \%$. Moreover, in some cases, the increase in the DG penetration level corresponds to the increase in the losses and load consumption. Consequently, it becomes necessary to seek for more balanced solutions, with reduced energy consumption and high DG penetration levels, as the provided by the multiobjective approach. At the end, the choice of a solution will depend on technical aspects, regulatory frameworks and commercial strategies. For example, the incentives the DNO receives to allow more DG connections, the economic impacts of energy efficiency, the energy cost and the operational costs of the ANM (i.e. the cost of loss of opportunity derived from energy curtailment, the cost of reactive power compensation and the depreciation of voltage regulation devices).

\section{Acknowledgment}

This work was supported by São Paulo Research Foundation (FAPESP) under grant: 2015/12911-3.

\section{References}

[1] Hallberg, P.: 'Active distribution system management a key tool for the smooth integration of distributed generation' (Eurelectric, 2013)

[2] Dent, C.J., Ochoa, L.F., Harrison, G.P.: 'Network distributed generation capacity analysis using OPF with voltage step constraints', IEEE Trans. Power Syst., 2010, 25, (1), pp. 296-304

[3] Vovos, P.N., Bialek, J.W.: 'Direct incorporation of fault level constraints in optimal power flow as a tool for network capacity analysis', IEEE Trans. Power Syst., 2005, 20, (4), pp. 2125-2134

[4] Keane, A., O'Malley, M.: 'Optimal utilization of distribution networks for energy harvesting', IEEE Trans. Power Syst., 2007, 22, (1), pp. 467-475

[5] AlKaabi, S.S., Khadkikar, V., Zeineldin, H.H.: 'Incorporating PV inverter control schemes for planning active distribution networks', IEEE Trans. Sustain. Energy, 2015, 6, (4), pp. 1224-1233

[6] Siano, P., Chen, P., Chen, Z., et al.: 'Evaluating maximum wind energy exploitation in active distribution networks', IET Gener. Transm. Distrib., 2010, 4, (5), pp. 598-608 
[7] Ochoa, L.F., Dent, C.J., Harrison, G.P.: 'Distribution network capacity assessment: variable DG and active networks', IEEE Trans. Power Syst., 2010, 25, (1), pp. 87-95

[8] Capitanescu, F., Ochoa, L.F., Margossian, H., et al.: 'Assessing the potential of network reconfiguration to improve distributed generation hosting capacity in active distribution systems', IEEE Trans. Power Syst., 2015, 30, (1), pp. 346-356

[9] Wang, S., Chen, S., Ge, L., et al.: 'Distributed generation hosting capacity evaluation for distribution systems considering the robust optimal operation of OLTC and SVC', IEEE Trans. Sustain. Energy, 2016, 7, (3), pp. 1111-1123

[10] Zio, E., Delfanti, M., Giorgi, L., et al.: 'Monte carlo simulation-based probabilistic assessment of DG penetration in medium voltage distribution networks', Int. J. Electr. Power Energy Syst, 2015, 64, pp. 852-860. Available at http://www.sciencedirect.com/science/article/pii/ S0142061514005304

[11] Cohon, J.L.: 'Multiobjective programming and planning' (Academic Press, USA, 1978)

[12] Rizy, D.T., Li, H., Li, F., et al.: 'Impacts of varying penetration of distributed resources with and without volt/var control: case study of varying load types'. 2011 IEEE Power and Energy Society General Meeting, 2011, pp. 1-7

[13] Quijano, D.A., Feltrin, A.P.: 'Assessment of conservation voltage reduction effects in networks with distributed generators'. 2015 IEEE PES Innovative Smart Grid Technologies Latin America (ISGT LATAM), 2015, pp. 393-398

[14] Padilha Feltrin, A., Rodezno, D.A.Q., Mantovani, J.R.S.: 'Volt-var multiobjective optimization to peak-load relief and energy efficiency in distribution networks', IEEE Trans. Power Deliv., 2015, 30, (2), pp. 618-626

[15] Wang, Z., Chen, B., Wang, J., et al.: 'Stochastic DG placement for conservation voltage reduction based on multiple replications procedure', IEEE Trans. Power Deliv., 2015, 30, (3), pp. 1039-1047

[16] Singh, D., Misra, R.K., Singh, D.: 'Effect of load models in distributed generation planning', IEEE Trans. Power Syst., 2007, 22, (4), pp. 2204-2212

[17] Wang, Z., Wang, J., Chen, B., et al.: 'MPC-based voltage/var optimization for distribution circuits with distributed generators and exponential load models', IEEE Trans. Smart Grid, 2014, 5, (5), pp. 2412-2420

[18] Singh, R., Tuffner, F., Fuller, J., et al.: 'Effects of distributed energy resources on conservation voltage reduction (CVR)'. In: 2011 IEEE Power and Energy Society General Meeting, 2011, pp. 1-7

[19] Ellis, A., Nelson, R., Engeln, E.V., et al.: 'Reactive power interconnection requirements for $P V$ and wind plants - recommendations to NERC' (Albuquerque, NM, Sandia National Laboratories, 2012). SAND2012-1098
[20] IEEE SCC 21: 'IEEE standard conformance test procedures for equipment interconnecting distributed resources with electric power systems amendment 1', IEEE Std 15471a-2015 (amendment to IEEE Std 15471-2005), 2015, pp. 1-27

[21] Atwa, Y.M., El Saadany, E.F., Salama, M.M.A., et al.: 'Optimal renewable resources mix for distribution system energy loss minimization', IEEE Trans. Power Syst., 2010, 25, (1), pp. 360-370

[22] Soroudi, A., Amraee, T. 'Decision making under uncertainty in energy systems: state of the art', Renew. Sustain. Energy Rev., 2013, 28, pp. 376-384. Available at http://www.sciencedirect.com/science/article/pii/ S1364032113005790

[23] Birge, J.R., Louveaux, F.: 'Introduction to stochastic programming' (Springer, USA, 1978, 2nd edn.)

[24] Baran, M.E., Wu, F.F.: 'Network reconfiguration in distribution systems for loss reduction and load balancing', IEEE Trans. Power Deliv., 1989, 4, (2), pp. $1401-1407$

[25] Korunović, L.M., Sterpu, S., Djokić, S., et al.: 'Processing of load parameter based on existing load models'. 2012 3rd IEEE PES Innovative Smart Grid Technologies Europe (ISGT Europe), 2012, pp. 1-6

[26] Heitsch, H., Römisch, W.: 'Scenario reduction algorithms in stochastic programming', Comput. Optim. Appl., 2003, 24, (2), pp. 187-206. Available at http://dx.doi.org/10.1023/A:1021805924152

[27] Slyke, R.M.V., Wets, R.: 'L-shaped linear programs with applications to optimal control and stochastic programming', SIAM J. Appl. Math., 1969, 17, (4), pp. 638-663

[28] Birge, J.R., Louveaux, F.V.: 'A multicut algorithm for two-stage stochastic linear programs', Eur J. Oper Res., 1988, 34, (3), pp. 384-392. Available at http://www.sciencedirect.com/science/article/pii/0377221788901592

[29] Crainic, T.G., Hewitt, M., Rei, W.: 'Partial Benders decomposition strategies for two-stage stochastic integer programs' (Publication, Centre interuniversitaire de recherche sur les réseaux d'entreprise, la logistique et le transport, Université de Montréal, Montréal, QC, Canada, 2016)

[30] IBM: 'IBM ILOG AMPL - User's guide', 2010. Available at http://ampl.com/ BOOKLETS/amplcplex122userguide.pdf

[31] IEEE PES: 'Distribution test feeders'. Available at http:/ ewh.iee.org/soc/pes/dsacom/testfeeders/ 